\title{
String-Generated Gravity Models
}

\author{
David G. Boulware \\ Department of Physics, University of Washington, Seattle, Washington 98195 \\ and \\ S. Deser \\ Department of Physics, Brandeis University, Waltham, Massachusetts 02254
}

(Received 17 October 1985)

\begin{abstract}
Expansion of supersymmetric string theory suggests that the leading quadratic curvature correction to the Einstein action is the Gauss-Bonnet invariant. We show that this model has both flat and anti-de Sitter space as solutions, but that the cosmological branch is unstable, because the graviton becomes a ghost there: The theory solves its own cosmological problem. The general static spherically symmetric solution is exhibited; it is asymptotically Schwarzschild. The sign of the Gauss-Bonnet coefficient determines whether there is a normal event horizon (for the stringgenerated sign) or a naked singularity. We discuss the effects of higher-curvature corrections and of an explicit cosmological term on stability.
\end{abstract}

PACS numbers: $12.25 .+\mathrm{e}, 04.20 . \mathrm{Cv}, 04.50 .+\mathrm{h}, 04.65 .+\mathrm{e}$

Classical Einstein theory is the low-frequency limit of quantum gravity. Although the correct quantum theory is not yet known, recent developments in string theory ${ }^{1}$ suggest that it is a promising candidate. Its slope expansion yields, in addition to the Einstein action, corrections quadratic and higher in the curvature. Of these, the quadratic term is of particular importance because it is the leading one and can affect the graviton excitation spectrum near flat space. If, like the string itself, its slope expansion is to be ghost free, the quadratic term, if any, must be the Gauss-Bonnet combination, which was first proposed ${ }^{2}$ for this reason and appears to be present ${ }^{3}$ in the heterotic string. ${ }^{4} \mathrm{~A}$ separate argument in its favor lies in the fact that the new gravitational Chern-Simons term necessary for anomaly freedom ${ }^{1}$ of $D=10$ supergravity requires the presence of the Gauss-Bonnet term for supersymmetry,${ }^{5}$ and, conversely, supersymmetrizing the Gauss-Bonnet term requires the Chern-Simons term. ${ }^{6}$

We propose here to analyze the properties of the effective gravity theory characterized by the Einstein plus Gauss-Bonnet terms, in order to see whether any qualitative properties of Einstein gravity are altered. We shall conclude that the Gauss-Bonnet combination, unlike any other quadratic terms, leads to a viable theory and that even the sign of its coefficient must be that dictated by the string expansion. This result is all the more remarkable because we shall see that there is an intrinsic cosmological-constant problem in all higher-curvature theories, which this particular model solves in an elegant way.

In addition to the asymptotically flat solutions, there is a cosmological, asymptotically anti-de Sitter branch. Such branches are a generic feature of actions with two or more curvature terms not involving explicit derivatives plus any number with derivatives. However, these solutions turn out to be intrinsically unstable: The graviton excitations about this background are ghosts, as is also confirmed by positive-energy sources leading to negative-mass Schwarzschild-anti-de Sitter solutions. We shall also discuss this branch in the presence of higher-order curvature corrections and of an explicit cosmological term, with qualitatively similar conclusions.

The action we consider has the form

$$
I=I_{1}+I_{2}=\int d^{D} x \sqrt{-g}\left[R / \kappa+\alpha\left(R_{\mu \nu \alpha \beta} R^{\mu \nu \alpha \beta}-4 R_{\mu \nu} R^{\mu \nu}+R^{2}\right)\right],
$$

with the convention $R_{\nu \alpha \beta}^{\mu} \sim+\Gamma_{\nu \beta, \alpha}^{\mu} \cdots, R_{\nu \beta} \equiv R_{\nu \mu \beta}^{\mu}, R \equiv R_{\nu}^{\nu}$, and signature $(-,+, \cdots,+)$. The action is in a $D \equiv n+2$ dimensional space-time. In units of $\kappa \equiv 4\left(\int d \Omega_{n}\right) G$, where $\int d \Omega_{n}$ is the area of a unit $n$-sphere, Newton's constant $G$ and $\kappa$ have dimensions $L^{D-3} / M$, while the expansion parameter $\kappa \alpha$ has dimension $L^{2}$, and the cosmological constant $\kappa \Lambda$ always has dimension $L^{-2}$. For $D=4$, the Gauss-Bonnet term $I_{2}$ is a topological invariant, and the only quadratic invariants with nonvanishing variations there are $R_{\mu \nu} R^{\mu \nu}$ and $R^{2}$. These will in general lead to ghosts near flat space $\left(h_{\mu \nu} \equiv g_{\mu \nu}-\eta_{\mu \nu}<<1\right)$ since their metric variations involve fourth time derivatives. By contrast, $I_{2}$ only contributes terms quadratic in $h_{\mu \nu}$ in the field equations, which read

$$
\begin{aligned}
& 0=\delta I / \delta g_{\mu \nu}=-G^{\mu \nu} / \kappa+\alpha T^{\mu \nu}, \\
& T_{\mu \nu}=\frac{1}{2} g_{\mu \nu}\left(R_{\alpha \beta \gamma \delta} R^{\alpha \beta \gamma \delta}-4 R_{\alpha \beta} R^{\alpha \beta}+R^{2}\right)-2 R R_{\mu \nu}+4 R_{\mu \alpha} R^{\alpha}{ }_{\nu}+4 R_{\alpha \beta} R^{\alpha}{ }_{\mu}{ }^{\beta}{ }_{\nu}-2 R_{\mu \alpha \beta \gamma} R_{\nu}{ }^{\alpha \beta \gamma} .
\end{aligned}
$$


It is easy to see that $T_{00}$ is of indefinite sign as it stands. In Gaussian normal coordinates $\left(g_{0 \mu}=\eta_{0 \mu}\right)$,

$$
T_{00}=\frac{1}{4}\left(R_{00}+R_{i}^{i}\right)^{2}+\frac{1}{4} R^{i j k l} R_{i j k l}-R_{j}^{i j k} R_{i l k}{ }^{l} .
$$

It is useful to write the action (1) in terms of forms or vielbeins, ${ }^{7,8}$

$$
\begin{aligned}
& I=\int\left\{\epsilon^{a_{1} \cdots a_{n}} R_{a_{1} a_{2}}\left[\omega_{a_{3}} \omega_{a_{4}}+\alpha \kappa n(n-1) R_{a_{3} a_{4}}\right] \omega_{a_{5}} \cdots \omega_{a_{D}}\right\}(\kappa n !)^{-1}, \\
& \text { where } \quad R_{a b}=d \omega_{a b}+\omega_{a}^{c} \omega_{c b}, \quad d \omega^{a}+\omega^{a}{ }_{b} \omega^{b}=0, \quad \omega^{a} \\
& =e^{a}{ }_{\mu} d x^{\mu} \text {, } \\
& \epsilon^{a_{1} \ldots a_{p} e_{1} \ldots e_{D-p}} \epsilon_{a_{1}^{\prime} \ldots a_{p}^{\prime} e_{1} \ldots e_{D-p}} \\
& =-(D-p) ! \delta_{a_{1}^{\prime}}^{a} \ldots a_{p}^{\prime \prime} \\
& 2 \kappa(n-1)(n-2) \mathscr{G}^{a b} \\
& =-\delta_{b_{1}}^{a_{1} \ldots a_{5}} R_{a_{2} a_{3}}^{b_{2} b_{3}}\left(\delta_{a_{4}}^{b_{4}} \delta_{a_{5}}^{b_{5}}+\frac{1}{2} \kappa \tilde{\alpha} R_{a_{4} a_{5}}^{b_{4} b_{5}}\right) \\
& =0 \text {. }
\end{aligned}
$$
upper and lower indices. An explicit cosmological term may be included as

$$
I_{\Lambda}=(\Lambda / D !) \int \epsilon^{e_{1} \cdots e_{D}} \omega_{e_{1}} \cdots \omega_{e_{D}} .
$$

The field equations corresponding to (3) are simply obtained by varying the basic one-forms $\omega^{a}$ because the variations of the connection one-forms yield a total derivative as long as the torsion vanishes. [These results are readily obtained by use of the relations $d \omega^{a}+\omega_{b}^{a} \omega^{b}=0=d R_{b}^{a}+\omega_{c}^{a} R_{b}^{c}+R^{a}{ }_{c} \omega_{b}^{c}$ ]. In particular, this means that one may regard (3) as being essentially in Palatini form with $\omega^{a}=e^{a}{ }_{\mu} d x^{\mu}$ and $\omega^{a}{ }_{b}=e^{a \nu} e_{b \nu ; \mu} d x^{\mu}$ as independent variables; this will be useful for an eventual canonical formulation of the theory. In this connection, we also note that despite being quadratic in curvature, the action depends only on first time derivatives; hence the number of degrees of freedom is the same as in Einstein gravity, and only the definition of the canonical momentum will change.

They are solved by the maximally symmetric spaces

$$
R_{c d}^{a b}=\lambda\left(\delta_{c}^{a} \delta_{d}^{b}-\delta_{d}^{a} \delta_{c}^{b}\right), \quad \lambda=0,-(\tilde{\alpha} \kappa)^{-1},
$$

where $\tilde{\alpha}=\alpha(n-1)(n-2)$; this follows because $\mathscr{G} a b$ reduces to $-\eta^{a b} \lambda(1+\tilde{\alpha} \lambda \kappa) / \kappa$. With our conventions, positive $\lambda$ (as dictated by string theor ${ }^{2,3}$ ) corresponds to anti-de Sitter space.

Next consider small variations about these backgrounds. Near flat space, we simply recover the ordinary graviton,

$$
0=\delta G_{b}^{a}=\left\{\bar{e}^{a \nu} \bar{e}_{c}{ }^{\lambda} \delta_{b}^{d}-\frac{1}{2} \delta_{b}^{a} \bar{e}^{d \nu} \bar{e}_{c}{ }^{\lambda}\right\} \delta R_{d \nu \lambda}^{c},
$$

where the explicit $\bar{e}$ 's are those of Minkowski space. There is no contribution from the Gauss-Bonnet term because the background curvature vanishes. Near anti-de Sitter space, however, the background curvature does not vanish and the variation is more delicate; there will be contributions proportional to curvatures times $h$ as well as second derivatives of $h$. We obtain from (5)

$$
\delta \mathscr{G}^{a}{ }_{\mu}=(1+2 \tilde{\alpha} \lambda \kappa) \delta G^{a}{ }_{\mu}+(\lambda n / 2)\left(\delta e^{a}{ }_{\mu}-\bar{e}^{a}{ }_{\mu} \bar{e}_{b}{ }^{\nu} \delta e^{b}{ }_{\nu}\right)[n-1+\tilde{\alpha} \lambda \kappa(n-3)],
$$

where the vielbeins are those of anti-de Sitter space. Since $\tilde{\alpha} \lambda \kappa=-1$, this becomes

$$
\begin{aligned}
& -\delta G_{\mu}^{a}+\lambda n\left(\delta e^{a}{ }_{\mu}-\bar{e}^{a}{ }_{\mu} \bar{e}_{b}{ }^{\nu} \delta e_{\nu}^{b}\right) \\
& \quad=0=-\delta G_{\mu \nu}+\lambda(n+1)\left(h_{\mu \nu}-\frac{1}{2} \bar{g}_{\mu \nu} h_{\lambda}{ }^{\lambda}\right)
\end{aligned}
$$

in vielbein and metric form, respectively. Note that the Gauss-Bonnet contribution in (7a) is precisely of the $\delta G^{a}{ }_{\mu}$ form but of opposite sign, reflecting the instability of anti-de Sitter space. The sign change in the field equation reflects a sign change in the second variation of the effective action; this implies that relative to anti-de Sitter space the kinetic terms have the opposite sign from that of the Minkowski-space kinetic terms. The derivative part of the variation of the Gauss-Bonnet term must be proportional to $\delta G^{a}{ }_{\mu}$ since this is the only second-derivative combination which can satisfy the Bianchi identity, while the nonderivative terms in (7) are needed to assure that $\delta \mathscr{G}^{\mu \nu}$

is covariantly conserved relative to the background metric. The complete $\delta \mathscr{G}^{\mu}{ }_{a}$ is essentially the (background) Lichnerowicz d'Alembertian acting on the metric or vielbein variation and it is easy to verify that $\bar{\nabla}_{\mu} \delta \mathscr{G}^{\mu}{ }_{\nu}=0$.

The sign change above arises as follows: The background solutions are obtained by solving $f(\lambda)$ $\equiv \lambda+\kappa \tilde{\alpha} \lambda^{2}=0$. The variations involve the slope $f^{\prime}(\lambda)$ of the parabola at the roots, $\lambda=0,-(\kappa \tilde{\alpha})^{-1}$. The slope is, of course, negative at the smaller intercept $-(\kappa \tilde{\alpha})^{-1}$; it is this slope which determines the sign of $\delta^{2} I$.

Let us compare the cosmological excitations (7b) to those of Einstein gravity with a ghost (negative metric) graviton,

$$
I=-\kappa^{-1} \int d^{D} x \sqrt{-g}[R+\Lambda \kappa]
$$

the field equation for which is $G_{\mu \nu}=\frac{1}{2} \Lambda \kappa g_{\mu \nu}$ [so that 
$\lambda=\Lambda \kappa / n(n+1)]$; its variation is precisely (7b). Thus, small excitations near the anti-de Sitter branch of the Einstein plus Gauss-Bonnet theory are precisely those of ghost cosmological Einstein gravity. As we shall see, the ghost nature is also manifested in the fact that a positive-energy matter source $T^{\mu \nu}$ leads to a negative-mass Schwarzschild-anti-de Sitter solution in which test particles are repelled.

We now derive the general spherically symmetric solutions of our model; just as in the Einstein theory their asymptotic forms also provide the leading correction to the backgrounds at infinity. (We remark that, by spherical symmetry, neither the antisymmetric tensor field nor the Chern-Simons terms which are also present in the string expansion can contribute here or alter the anti-de Sitter background solution). We choose our interval in Schwarzschild gauge, and assume time independence, although this should be unnecessary, since we expect Birkhoff's theorem to hold as there is only a tensor excitation. The calculations of the various curvature components are straightforward, and the problem may be further simplified by working directly in the action. For general static geometries the action may be a priori predicted (and explicitly verified) to have the form

$$
I \rightarrow I_{s}=\int d^{n+1} x N \sqrt{g_{s}}\left[R \kappa^{n-1}+\alpha\left(R^{\mu \nu \lambda \sigma} R_{\mu \nu \lambda \sigma}-4 R^{\mu \nu} R_{\mu \nu}+R^{2}\right)\right]_{s},
$$

where $N \equiv\left(-g_{00}\right)^{1 / 2}$ and all curvatures are intrinsic to the spatial section as indicated by the subscript $s$; this is because $N$ continues, in this model as for Einstein, to be the Lagrange multiplier for the $\mathscr{G}^{00}$ constraint. In our gauge, $d s^{2}=-e^{2 \phi} d t^{2}+e^{2 \lambda} d r^{2}+r^{2} d \Omega_{n}^{2}, I_{s}$ reduces to the amazingly simple form

$$
I_{s}=n(4 G)^{-1} \int d r e^{\phi+\lambda}\left[r^{n+1} \psi(1+\kappa \tilde{\alpha} \psi)\right]^{\prime}, \quad \psi=r^{-2}\left(1-e^{-2 \lambda}\right) .
$$

The corresponding field equations integrate to

$$
\psi(1+\kappa \tilde{\alpha} \psi)=2 M G / r^{n+1}, \quad e^{\phi+\lambda}=1 .
$$

The constant $M$ is, of course, the mass with the sign chosen to correspond to a positive semidefinite matter energy density. For $M=0$, we recover flat $(\psi=0)$ and anti-de Sitter $\left[\psi=-(\tilde{\alpha} \kappa)^{-1}\right]$ spaces, respectively. In general we have

$$
-g_{00}=e^{2 \phi}=e^{-2 \lambda}=e^{-2 \lambda}=1+\left(r^{2} / 2 \tilde{\alpha} \kappa\right)\left\{1 \mp\left[1+\left(8 G M \tilde{\alpha} \kappa / r^{n+1}\right)\right]^{1 / 2}\right\},
$$

the asymptotic behavior of which is

$$
-g_{00} \sim 1-\left(2 G M / r^{n-1}\right) \text { or }\left[1+\left(r^{2} / \tilde{\alpha} \kappa\right)\right]+\left(2 G M / r^{n-1}\right),
$$

i.e., Schwarzschild with positive gravitational mass or Schwarzschild-de Sitter with negative gravitational mass, with the standard energy definition in background anti-de Sitter space. ${ }^{9}$ This illustrates our earlier statements about the instability of the anti-de Sitter branch. Note that $\tilde{\alpha}$ has disappeared from the leading asymptotic corrections to the respective backgrounds for both branches. However, $\tilde{\alpha}$ defines the background in the anti-de Sitter branch, which shows that the solutions of the effective theory do not always reduce to Einstein spaces asymptotically; the Einstein, "long wavelength," limit is only recovered for distances smaller than the length defined by $\left(\tilde{\alpha}_{\kappa}\right)^{1 / 2}$.

If there is also an explicit cosmological term in the action, representing eventual supersymmetry breakdown, the solution is still simple. A term $\Lambda \sqrt{-g}$ merely adds the constant $\Lambda / n(n+1)$ to the left-hand side of the first equation of (11), with the result that the effective cosmological constant is

$$
\Lambda_{\text {eff }}=\left[n(n+1) / 2 \tilde{\alpha} \kappa^{2}\right]\left(1 \mp\left\{1-\left[4 \tilde{\alpha} \kappa^{2} \Lambda / n(n+1)\right]^{1 / 2}\right\}\right) ;
$$

the larger value will be unstable, the lower one stable. Note that if $4 \Lambda \kappa^{2} \tilde{\alpha}$ is greater than $n(n+1)$ the theory is undefined. The corresponding form of (12) has the same extra term in its square root as that in (13).

The singularity properties of the metric (12) may easily be obtained. We consider only the stable asymptotically flat regime, merely noting that the anti-de Sitter branch $(\alpha>0)$ has a naked singularity at the origin but no horizons. Our first observation is that the sign of $\alpha$ is critical: The positive sign dictated by the string expansion is the only physically sensible one. For if $\alpha$ were negative, the metric would become imaginary at $r^{n+1}=8|\tilde{\alpha}|_{\kappa} M G$. This is a real singularity, since the curvature invariants have branch singularities there; furthermore the singularity is naked when, as is possible, it occurs outside the event horizon, $g_{00}\left(r_{H}\right)=0$. For $\alpha>0$ there is in general always one and only one horizon; $g_{00}$ vanishes at values $r_{H}$ such that $r_{H}^{D-3}+\tilde{\alpha} \kappa r_{H}^{D-5}=2 G M$. For $D>5$, the left-hand 
side ranges monotonically from zero to infinity with $r_{H}$, so that there is exactly one solution. For $D=5$, the existence of a horizon depends on whether $2 G M-\tilde{\alpha} \kappa$ is positive, which is the case for macroscopic masses. Otherwise, there is a naked conical singularity at the origin. (That $D=5$ is special can be understood from the appearance of a factor $D-5$ in the variation of $T^{\mu \nu}$.) While the value of $r_{H}$ at which the horizon occurs differs from the Einstein one, the qualitative properties of the geometry are identical. The usual Kruskal completion procedure goes through just as in Schwarzschild, and Hawking radiation will still be present.

Although Einstein $\left(G_{\mu \nu}=0\right)$ spaces are not generally solutions of (2), it is interesting to mention one special case which is, because it underlines the fact that the Gauss-Bonnet term does not affect the propagator, and in particular does not distort plane-wave solutions. The plane-fronted parallel-ray metric, ${ }^{10}$ although globally singular, is the nearest thing to a graviton in the Einstein theory; it remains a solution here. The interval $d s^{2}=2 H\left(u, x_{\perp}\right) d u^{2}+2 d u d v+d \mathbf{x}_{\perp}^{2}$ obeys $G_{\mu \nu}=0$ iff $\nabla_{\perp}^{2} H=0$. Its only nonvanishing curvature component is $R^{v}{ }_{i u l}=\partial_{i l}^{2} H$, which implies the vanishing of $R_{\mu \alpha \beta \gamma} R_{\nu}{ }^{\alpha \beta \gamma}$ and hence of $\tilde{\alpha} T_{\mu \nu}$ since all other terms in (2) vanish in Einstein geometries.

We conclude with some remarks about possible higher-curvature corrections in the effective theory given by the string expression.

For fixed $D$, there is only a limited number of Gauss-Bonnet $(\epsilon \epsilon R \cdots R e \cdots)$ terms (e.g., through $R^{4}$ for $D=9$ or 10 ), although there is no limit to derivative powers $\sim \epsilon \epsilon R \cdots \square^{k} R e \cdots e$. There seems in any case to be no a priori reason why only these terms should appear, beyond the quadratic one, and one should consider also generic $R_{\mu \nu \alpha \rho}^{3}$, etc., corrections. No higher-order terms can spoil the asymptotically flat branch, since they do not contribute to small excitations there. The only dangerous terms are of the form $R \square^{p} R$, but note that, by the Bianchi identities, $\epsilon \epsilon R \square^{p} R$ is actually cubic in $R$. However, this is not the case for the anti-de Sitter branch: Generic contributions of the form $R^{p}$ will yield $\sim \nabla^{2}\left(R^{p-1}\right)$ terms in the field equations and so lead to variations of the form $R^{p-2} \nabla^{2} \delta R \sim \square \square h$. Gratifyingly, whatever the contributions to $\sim \square h$ terms, the $\square^{2} h$ ghosts will exclude the anti-de Sitter branch by stability arguments. More complicated is the effect of higher-order Gauss-Bonnet terms. These will not yield $\square^{2} h$ effects, but will contribute to $\delta G_{\mu \alpha}$. Following our discussion of the quadratic case, $\lambda$ will be determined by a polynomial equation, $f(\lambda)$ $=\lambda\left(1+\tilde{\alpha} \kappa \lambda+\gamma_{2} \lambda^{2} \kappa+\cdots\right)=0$, where the $\gamma_{i}$ are essentially the coefficients of the higher terms. To the extent that there are real roots, the slopes at the intercepts will have alternating signs and therefore the per- turbations $\delta G_{\mu \alpha}$ will appear with both signs. (For example, a cubic Gauss-Bonnet term with $\gamma_{2}>\tilde{\alpha}^{2} \kappa / 4$ yields no real roots and hence no cosmological branch. It would be interesting if this "prediction" were verified.) So it is conceivable that there would be stable anti-de Sitter sectors in the (unlikely) event that the only corrections are of the Gauss-Bonnet type with (un-) suitable coefficients. If, in addition, there is an explicit cosmological term, the situation changes. Now we have $f(\lambda)-\Lambda=0$ to solve, which cannot include a flat background. Consequently, the ghost problem could make all solutions unstable for generic $R^{n}$ corrections, which is perhaps too neat a disposal of the cosmological-constant problem! Presumably the string either avoids the explicit $\Lambda$, or has only Gauss-Bonnet corrections with suitable coefficients. We also note that the scalar field which we have neglected here will play a role in these questions.

What is the relation between the instability we noted in the cosmological branch and the form of the total energy for our model? If, as expected, ${ }^{5,6}$ a supersymmetric generalization exists (presumably only for $\alpha>0$ ), it may or may not have still higher-curvature terms, but will in any case contain antisymmetric tensor and scalar fields. By general classical supersymmetry arguments, ${ }^{11}$ the bosonic sector will have positive energy, if and only if it is free of ghost fermions. ${ }^{12}$ (For example in $D=4, R+R_{\mu \nu}^{2}+R^{2}$ theories are supersymmetrizable but, because they have ghosts, they are unstable even near flat space.) What we expect here is that the total energy will be positive for the asymptotically flat solutions, but not for the anti-de Sitter branch.

We have seen that the leading string-generated correction to Einstein gravity defines a theory with a number of striking properties. In addition to being ghost free near flat space, the model cures its own cosmological-constant problem in an elegant fashion, through just the sort of negative-energy instability in the de Sitter sector which does not occur in the normal Einstein plus cosmological term theory, or with generic quadratic curvature terms. Even in the presence of an explicit $\Lambda$ term, it prefers the lower effective cosmological value. The sign of the correction term is just that one which avoids singularities in the solutions. For it, the "Schwarzschild" solution is perfectly sensible, with the usual event horizon structure. We also noted that cubic or higher corrections will only reinforce the unique physically acceptable nature of the asymptotically flat branch, since they imply unavoidable ghosts in the anti-de Sitter branch. However, it is too early to speculate on these higher-order questions. There are sufficiently many unresolved matters here: First is, of course, the effect of compactification on our $D>4$ dimensional constructions, and of scalar and antisymmetric tensor fields which are 
present in the supergravity action, together with the Chern-Simons term. It would also be useful to construct the canonical formulation for this "already Palatini" system, and to build up more extensive understanding of the space of allowed classical geometries beyond the plane-wave Einstein solution given here.

This work was supported in part by the U.S. Department of Energy under Contract No. DE-AC0681 ER40048 and by the National Science Foundation under Grant No. PHY 82-01094. One of us (D.G.B.) thanks the Aspen Center for Theoretical Physics. After our paper was completed, we were informed by J. T. Wheeler that he had independently obtained the asymptotically flat branch of the "Schwarzschild" solution (12a).

${ }^{1}$ M. B. Green and J. H. Schwarz, Phys. Lett. 149B, 177 (1984), and 151B, 21 (1985).

2B. Zwiebach, Phys. Lett. 156B, 315 (1985), and in Symposium on Anomalies, edited by W. A. Bardeen and A. R. White (World Scientific, Singapore, 1985).

${ }^{3}$ R. Nepomechie, Phys. Rev. D (to be published).

${ }^{4}$ C. G. Callan, E. Martinec, M. J. Perry, and D. Friedan (to be published); A. Sen, Fermilab Report No. Pub-85/81T (to be published); D. J. Gross, J. Harvey, E. Martinec, and R. Rohm, Phys. Rev. Lett. 54, 502 (1984), and Nucl.
Phys. B 256, 253 (1985), and to be published. See also E. S. Fradkin and A. A. Tseytlin, Lebedev Institute Report No: N261, 1984 (to be published).

${ }^{5} \mathrm{~L}$. Romans and N. Warner, California Institute of Technology Report No. 88-1291 (to be published); S. Cecotti et al., CERN Report No. TH4253/85, 1985 (to be published).

${ }^{6} \mathrm{~S}$. Deser, in Proceedings of the Workshop on Supersymmetry and its Applications, Cambridge, 1985 (Cambridge Univ. Press, Cambridge, Mass., to be published).

${ }^{7}$ B. Zumino, University of California, Berkeley, Report No. UCB-PTH-85/13 (to be published); see also D. Lovelock, J. Math. Phys. 12, 498 (1971).

${ }^{8}$ S. MacDowell and F. Mansouri, Phys. Rev. Lett. 38, 739 (1977).

${ }^{9}$ L. Abbott and S. Deser, Nucl. Phys. B 195, 76 (1982).

${ }^{10} \mathrm{~J}$. Ehlers and W. Kundt, in Gravitation, edited by L. Witten (Wiley, New York, 1962).

${ }^{11} \mathrm{G}$. Horowitz and A. Strominger, Phys. Rev. D 27, 2793 (1983); S. Deser, Phys. Rev. D 27, 2805 (1983), and 30, 1392 (1984); C. Teitelboim, Phys. Rev. D 29, 2763 (1984); C. Hull, Commun. Math. Phys. 90, 545 (1983).

${ }^{12}$ D. G. Boulware and S. Deser, Phys. Rev. D 30, 707 (1984); D. G. Boulware, S. Deser, and K. S. Stelle, in "Quantum Field Theory and Quantum Statistics" (Hilger, Bristol, England, to be published).

${ }^{13} \mathrm{We}$ have not considered whether any semiclassical tunneling modifications will occur through the existence of two branches of solutions; this seems unlikely, given that they correspond to different boundary conditions and the values of the Euclidean action are quite different. 